\title{
Properties of Wave Motion in a Cylindrical Shell, Interacting with Viscous Liquid
}

\author{
Ismail Ibrahimovich Safarov', Muhsin Khudoyberdiyevich Teshaev², \\ Zafar Ixtiyorovich Boltayev ${ }^{2}$ \\ ${ }^{1}$ Tashkent Chemistry-Technological Institute, Tashkent, Republic of Uzbekistan \\ ${ }^{2}$ Bukhara Technological-Institute of Engineering, Bukhara, Republic of Uzbekistan \\ Email: safarov54@mail.ru
}

How to cite this paper: Safarov, I.I., Teshaev, M.K. and Boltayev, Z.I. (2018) Properties of Wave Motion in a Cylindrical Shell, Interacting with Viscous Liquid. Open Access Library Journal, 5: e4563. https://doi.org/10.4236/oalib.1104563

Received: April 2, 2018

Accepted: May 14, 2018

Published: May 17, 2018

Copyright $\odot 2018$ by authors and Open Access Library Inc.

This work is licensed under the Creative Commons Attribution International License (CC BY 4.0).

http://creativecommons.org/licenses/by/4.0/

(c) () Open Access

\begin{abstract}
The propagation of natural waves in a cylindrical shell (elastic or viscoelastic) that is in contact with a viscous liquid is considered. The problem reduces to solving spectral problems with a complex incoming parameter. The system of ordinary differential equations is solved numerically, using the method of orthogonal rotation of Godunov with a combination of the Muller method. The dissipative processes in the mechanical system are investigated. A mechanical effect is obtained that describes the intensive flow of mechanical energy.
\end{abstract}

\section{Subject Areas}

Continuum Mechanics

\section{Keywords}

The Cylindrical Shell, Viscous Liquid, Wave Process, Dissipative Non-Uniform, Wavy Motion

\section{Introduction}

Quantitative data and some qualitative analysis of the dispersion properties of normal waves in a cylinder with a liquid were carried out in [1] [2] [3] [4]. In [5] [6] [7] [8], data were obtained for cylinders filled with a liquid of solid and soft materials. It is studied in a rather wide range of frequencies and wave numbers. The effect of the wall thickness of the cylinder on the dispersion properties of normal waves in the range of variation of the relative internal radiation is from 0.3 to 0.99 [9] [10]. This article focuses on the dynamic behavior of a cylindrical shell (elastic or viscoelastic), which contains a viscous liquid. The problem of wave propagation in a filled or submerged liquid with a cylindrical shell is of great practical importance. The phenomenon of undulating fluid motion in elas- 
tic cylindrical shells has attracted the attention of many researchers [11]-[16]. In these papers, wave processes in an elastic cylindrical shell containing an ideal fluid are considered. Also, the refined classical shell equations, the average fluid flow (or gas) density, the influence of radial and longitudinal inertia forces are used. In [17] [18] [19], the laws of wave processes in an elastic shell with a viscous fluid are analyzed in the model of linear equations of hydrodynamics of a viscous compressible fluid. Unlike other systems, a cylindrical shell (elastic or viscoelastic) and liquid (ideal or viscous) are considered as an inhomogeneous dissipative mechanical system [20] [21] [22].

\section{Statement of the Problem}

An infinite length of deformable (viscoelastic) cylindrical shell of radius $\mathrm{R}$ with constant thickness $h_{0}$, density $\rho_{0}$, Poisson's ratio $v_{0}$, filled with a viscous fluid with density at equilibrium. Fluctuations of a shell under a load, the density of which is denoted $p_{1}, p_{2}, p_{n}$ respectively, can be described by following [11] [12] [14], equations:

$$
L \boldsymbol{u}-L_{0} \int_{0}^{t} R_{L}(t-\tau) L \boldsymbol{u}(\tau) \mathrm{d} \tau=\frac{\left(1-v_{0}^{2}\right)}{E_{0} h_{0}} \boldsymbol{p}+\rho_{0} \frac{\left(1-v_{0}^{2}\right)}{E_{0}}\left(\frac{\partial^{2} \boldsymbol{u}}{\partial t^{2}}\right),
$$

Here $\boldsymbol{u}=\boldsymbol{u}\left(u_{r}, u_{\theta}, u_{z}\right)$-displacement vector points of the middle surface of the shell and membranes for Kirchhoff-Love it has a dimension equal to three $\left(u_{r}=u ; u_{\theta}=v ; u_{z}=w\right)$, and to membranes such as the dimension of Timoshenko $\boldsymbol{u}$ is five. Here, in addition to the axial, circumferential and normal movements added more angles of rotation normal to the middle surface in the axial and circumferential directions [22]; $\left\{\begin{array}{lll}u & v & w\end{array}\right\}^{\mathrm{T}}$-the displacement vector with axial, radial and circumferential components, respectively ("+" sign in front of $p_{n}$ and the sign "-" before the last component of the inertial member says that is considered positive motion towards the center of curvature); $R_{L}(t-\tau)$-the core of relaxation; $L_{0}$-instantaneous modulus of elasticity.

The amplitudes of the oscillations are considered small, which allows you to record the basic relations in the framework of the linear theory. The system of linear equations of motion of a viscous barotropic liquid can be written as [22]:

$$
\begin{aligned}
& \frac{\partial \vartheta}{\partial t}-v^{*} \Delta \vartheta+\frac{1}{\rho_{0}^{*}} \operatorname{grad} P-\frac{\nu^{*}}{3} \operatorname{grad} \operatorname{div} \vartheta=0 \\
& \frac{1}{\rho_{0}^{*}} \frac{\partial \rho^{*}}{\partial t}+\operatorname{div} \vartheta=0 ; \frac{\partial P}{\partial \rho^{*}}=a_{0}^{2}, a_{0}=\text { const. } \\
& \dot{u}_{z}=\vartheta_{z}, \dot{u}_{r}=\vartheta_{r}, \dot{u}_{\theta}=\vartheta_{\theta}, \\
& q_{z}=-p_{r z}, q_{r}=-p_{r}, q_{\theta}=-p_{r \theta} . \\
& p_{r z}=\mu^{*}\left(\frac{\partial \vartheta_{z}}{\partial r}+\frac{\partial \vartheta_{r}}{\partial z}\right) ; \\
& p_{r r}=-p+\lambda^{*}\left(\frac{\partial \vartheta_{r}}{\partial r}+\frac{\partial \vartheta_{z}}{\partial z}+\frac{\vartheta_{r}}{r}\right)+2 \mu^{*} \frac{\partial \vartheta_{r}}{\partial r} ; \\
& p_{r \theta}=\mu^{*}\left(\frac{1}{r} \frac{\partial \vartheta_{z}}{\partial \theta}+\frac{\partial \vartheta_{\theta}}{\partial r}-\frac{\vartheta_{\theta}}{r}\right) .
\end{aligned}
$$


Here, in the Equations (2) $\vartheta=\vartheta\left(\vartheta_{r}, \vartheta_{\theta}, \vartheta_{z}\right)$-the velocity vector of fluid particles; $\rho^{*}$ and $P$-disturbance density and fluid pressure; $\rho_{0}^{*}$ and $a_{0}$-density and sound velocity in the fluid at rest; $v^{*}, \mu^{*}$-kinematic and dynamic viscosity; for the second viscosity coefficient $\lambda^{*}$ accepted ratio $\lambda=-\frac{2}{3} \mu^{\bullet} ; p_{r z}, p_{r r}, p_{r \theta}-$ components of the stress tensor in the fluid. Equation (1), respectively, kinematic and dynamic boundary conditions, which, because of the thin-walled shell, we will meet on the middle surface $(r=R)$. Equations (1) and (2) is a closed system of relations hydro visco elastic cylindrical shell for containing a viscous compressible fluid. This is for shell obeying Kirchhoff-Love hypotheses. Be investigated joint shell and liquid fluctuations, harmonic of the axial coordinate $z$ and decay exponentially over time, or time-harmonic and damped with respect to $z$.

\section{Method of Solution}

We accept the integral terms in (1) small, then the function

$$
\boldsymbol{u}(\boldsymbol{r}, t)=\boldsymbol{u}_{1}(\boldsymbol{r}, t) \mathrm{e}^{-i \omega_{R} t}
$$

where $\psi(\boldsymbol{r}, t)$-slowly varying function of time, $\omega_{R}$-real constant. Next, using the procedure of freezing [23], then the integral-differential Equation (1) takes the form

$$
L\left[1-\Gamma^{C}\left(\omega_{R}\right)-i \Gamma^{S}\left(\omega_{R}\right)\right] \boldsymbol{u}_{1}=\frac{\left(1-v_{0}^{2}\right)}{E_{0} h_{0}} \boldsymbol{p}+\rho_{0} \frac{\left(1-v_{0}^{2}\right)}{E_{0}}\left(\frac{\partial^{2} \boldsymbol{u}_{1}}{\partial t^{2}}\right),
$$

where, for shell Kirchhoff-Love

$$
L=\left(\begin{array}{ccc}
\frac{\partial^{2}}{\partial x^{2}}+\frac{1-v_{0}}{2 R^{2}} \frac{\partial^{2}}{\partial \varphi^{2}} & \frac{1+v_{0}}{2 R} \frac{\partial^{2}}{\partial x \partial \varphi} & \frac{v_{0}}{R} \frac{\partial}{\partial x} \\
\frac{1+v_{0}}{2 R} \frac{\partial^{2}}{\partial x \partial \varphi} & \frac{1+v_{0}}{2}(1+4 a) \frac{\partial^{2}}{\partial x^{2}}+(1+a) \frac{\partial^{2}}{\partial \varphi^{2}} & \frac{1}{R^{2}} \frac{\partial}{\partial \varphi}-a\left(2-v_{0}\right) \frac{\partial^{3}}{\partial x^{2} \partial \varphi}-\frac{a}{R^{2}} \frac{\partial^{3}}{\partial \varphi^{3}} \\
\frac{v_{0}}{R} \frac{\partial}{\partial x} & \frac{1}{R^{2}} \frac{\partial}{\partial \varphi}-a\left(2-v_{0}\right) \frac{\partial^{3}}{\partial x^{2} \partial \varphi}-\frac{a}{R^{2}} \frac{\partial^{3}}{\partial \varphi^{3}} & \frac{1}{R^{2}}+a\left(\frac{\partial^{2}}{\partial x^{2}}+\frac{1}{R^{2}} \frac{\partial^{2}}{\partial \varphi^{2}}\right)^{2}
\end{array}\right),
$$

and sine Fourier transforms relaxation kernel material. As an example, the viscoelastic material take three parametric kernel relaxation $R_{L}(t)=A \mathrm{e}^{-\beta t} / t^{1-\alpha}$, $\rho$-material density shell; $E$-Young's modulus; $v-$ Poisson's ratio, $a=h^{2} / 12 R^{2}$ Let's move on to the dimensionless axial coordinate $\xi=x / R$ and multiply by $R^{2}$ system (3). The matrix of the resulting system will take the form

$$
L=\left(\begin{array}{ccc}
\frac{\partial^{2}}{\partial \xi^{2}}+\frac{1-v_{0}}{2} \frac{\partial^{2}}{\partial \varphi^{2}} & \frac{1+v_{0}}{2} \frac{\partial^{2}}{\partial \xi \partial \varphi} & v_{0} \frac{\partial}{\partial \xi} \\
\frac{1+v_{0}}{2} \frac{\partial^{2}}{\partial \xi \partial \varphi} & \frac{1-v_{0}}{2}(1+4 a) \frac{\partial^{2}}{\partial \xi^{2}}+(1+a) \frac{\partial^{2}}{\partial \varphi^{2}} & \frac{\partial}{\partial \varphi}-a\left(2-v_{0}\right) \frac{\partial^{3}}{\partial \xi^{2} \partial \varphi}-a \frac{\partial^{3}}{\partial \varphi^{3}} \\
v_{0} \frac{\partial}{\partial \xi} & \frac{\partial}{\partial \varphi}-a\left(2-v_{0}\right) \frac{\partial^{3}}{\partial \xi^{2} \partial \varphi}-\frac{a}{R^{2}} \frac{\partial^{3}}{\partial \varphi^{3}} & \frac{1}{R^{2}}+a\left(\frac{\partial^{2}}{\partial \xi^{2}}+\frac{1}{R^{2}} \frac{\partial^{2}}{\partial \varphi^{2}}\right)^{2}
\end{array}\right) .
$$

Expanding Equation (2) and (3) in coordinate form, it is easy to see that the 
relations (2) - (3) break up into independent boundary value problems:

\section{- Torsional vibrations:}

$$
\begin{aligned}
& \frac{\partial p_{r \theta}}{\partial r}+\frac{2 p_{r \theta}}{r}+\frac{\partial p_{\theta z}}{\partial z}=\rho_{0}^{*} \ddot{\vartheta}_{\theta}, \\
& p_{r \theta}=\mu^{*}\left(\frac{\partial \vartheta_{\theta}}{\partial r}-\frac{\vartheta_{\theta}}{r}\right), \quad p_{\theta z}=\mu^{*} \frac{\partial \vartheta_{\theta}}{\partial r}, \\
& r=R_{1}: \bar{G} h_{0} \frac{\partial^{2} u_{\theta}}{\partial z^{2}}-\left(\rho_{0} h \ddot{u}_{\theta} \pm \sigma_{\varphi r}\right)=0, \bar{G}=\frac{\bar{E}}{2\left(1+v_{0}\right)}, \\
& r=0: p_{r \theta}=0, \bar{E}=E_{0}\left(1-\Gamma^{C}\left(\omega_{R}\right)-i \Gamma^{S}\left(\omega_{R}\right)\right)
\end{aligned}
$$

\section{- Longitudinal transverse vibrations:}

$$
\begin{gathered}
\frac{\partial p_{r r}}{\partial r}+\frac{p_{r r}-p_{\theta \theta}}{r}+\frac{\partial p_{r z}}{\partial z}=\rho_{0}^{*} \frac{\partial \vartheta_{r}}{\partial t} \\
\frac{\partial p_{r z}}{\partial r}+\frac{p_{r z}}{r}+\frac{\partial p_{z z}}{\partial z}=\rho_{0}^{*} \frac{\partial \vartheta_{z}}{\partial t} \\
p_{r r}=-p+\lambda^{*} k_{\eta} \operatorname{div} \vartheta+2 \mu^{*} \frac{\partial \vartheta_{r}}{\partial r}, \\
p_{\theta \theta}=-p+\lambda^{*} \operatorname{div} \vartheta+2 \mu^{*} \frac{\vartheta_{r}}{r} \\
p_{z z}=-p+\lambda^{*} \operatorname{div} \vartheta+2 \mu^{*} \frac{\partial \vartheta_{z}}{\partial z} \\
\frac{\partial \rho^{*}}{\partial t}+\rho_{0} d i v=0, \operatorname{div} \vartheta=\frac{\partial \vartheta_{r}}{\partial r}+\frac{\vartheta_{r}}{r}+\frac{\partial \vartheta_{z}}{\partial z}, \frac{\partial p}{\partial \rho}=a_{0}^{2} \\
\left.r=\frac{\partial \vartheta_{r}}{\partial z}\right), \quad p_{r \theta}=\mu^{*}\left(\frac{1}{r} \frac{\partial \vartheta_{z}}{\partial \theta}+\frac{\partial \vartheta_{\theta}}{\partial r}+\frac{\vartheta_{r}}{r}\right) \\
\bar{D} \frac{\partial^{4} u_{r}}{\partial z^{4}}+\frac{\bar{C}}{R_{1}}\left(\frac{u_{r}}{R_{1}}+v_{0} \frac{\partial u_{z}}{\partial z}\right)+p_{r r}+\rho_{0} h_{0} \frac{\partial^{2} u_{r}}{\partial t^{2}}=0, \\
\bar{C}\left(\frac{\partial^{2} u_{r}}{\partial z^{2}}+\frac{v_{0}}{R} \frac{\partial u_{r}}{\partial z}\right)-\left(p_{r z} \pm \rho_{0} h_{0} \frac{\partial^{2} u_{z}}{\partial t^{2}}\right)=0,
\end{gathered}
$$

Let the wave process is periodic in $z$ and fades over time, then is given a real wave number $k$, and the complex frequency is the desired characteristic value. Solution of (2) - (6) for the major unknowns satisfying constraints imposed above the dependence on time and coordinates $z$, should be sought in the form [24]

$$
\begin{aligned}
& \left(p_{r r}, p_{r z}, p_{r \theta}, \boldsymbol{u}, \boldsymbol{\vartheta}\right)^{\mathrm{T}} \\
& =\sum_{m}\left(\sigma_{r m}(\xi, \varphi, t), \tau_{z m}(\xi, \varphi, t), \tau_{\varphi m}(\xi, \varphi, t), \boldsymbol{u}_{m}(\xi, \varphi, t), \boldsymbol{\vartheta}_{m}(\xi, \varphi, t)\right)^{\mathrm{T}},
\end{aligned}
$$

where $\boldsymbol{u}_{m}(\xi, \varphi, t)=\boldsymbol{u}_{m}\left\{U_{m}, V_{m}, W_{m}\right\}^{\mathrm{T}}, \vartheta(\xi, \varphi, t)=\vartheta_{m}\left\{\vartheta_{r m}, \vartheta_{\theta m}, \vartheta_{z m}\right\}^{\mathrm{T}}$.

Expressions (7) in the form

$$
\begin{aligned}
& \left(\sigma_{r m}(\xi, \varphi, t), \tau_{z m}(\xi, \varphi, t), \tau_{\varphi m}(\xi, \varphi, t)\right)^{\mathrm{T}} \\
& =\left(\sigma_{r} \cos (m \varphi), \tau_{z} \cos (m \varphi), \tau_{\varphi} \sin (m \varphi)\right)^{\mathrm{T}} \mathrm{e}^{i k z-i \omega t},
\end{aligned}
$$




$$
\begin{aligned}
& \left(\boldsymbol{u}_{m}(\xi, \phi, t), \vartheta_{m}(\xi, \varphi, t)\right)^{\mathrm{T}} \\
= & \left(U_{m} \cos (m \varphi), V_{m} \sin (m \varphi), W_{m} \cos (m \varphi),\right. \\
& \left.\vartheta_{r} \cos (m \varphi), \vartheta_{\theta} \cos (m \varphi), \vartheta_{z} \cos (m \varphi)\right)^{\mathrm{T}} \mathrm{e}^{i k z-i \omega t},
\end{aligned}
$$

where $\sigma_{r}, \tau_{z}, \tau_{\varphi}, U_{m}, V_{m}, W_{m}, \vartheta_{r}, \vartheta_{\theta}, \vartheta_{z}$-Amplitude integrated vector-function; $\kappa$-wavy number; $C$-phase velocity; $\omega$-complex frequency; $m$-circumferential wave number (the number of district-wave), takes values $m=1,2,3, \cdots$. When $m$ $=0$, happening Ax symmetrical vibrations. This approach allows you to seek a solution for every fixed value of the wave number of the district $m$ independently.

In this way $C, k, \omega$ it is well-known real and complex spectral parameters of the type of problem.

To elucidate their physical meaning consider two cases:

1) $\kappa=\kappa_{R} ; C=C_{R}+i C_{i}$, Then the solution of (5) has the form of a sine wave $\boldsymbol{x}$, whose amplitude decays over time;

2) $\kappa=\kappa_{R}+i \kappa_{I} ; C=C_{R}$, Then at each point $\boldsymbol{x}$ fluctuations established, but $\boldsymbol{x}$ attenuate. In the case of axially symmetric on the axis $r=0$ conditions must be satisfied conditions $p_{r \theta}=p_{r z}=0, \vartheta_{r}=0$. If the outer surface $\boldsymbol{\Gamma}=\boldsymbol{R}$ assumed stationary, then $u_{r}=u_{z}=u_{\varphi}=0$. The superposition of the solutions (8) forms an exponentially decaying over time the standing wave that describes the natural oscillations of a liquid and a cylindrical shell of finite length with boundary conditions. With infinite length sheath similarly specified type of movement (8) will be called private or free fluctuations. In the case of steady-state over time and fading coordinate the process, in contrast, is a well-known real rate of $\omega$, as desired be a complex wave number $k$. In contrast to their own, these fluctuations will be called the established. Actual values of the $\omega$ in the first case, and $k$, second frequency have the physical meaning of the process in time and the coordinate, respectively. Imaginary part-the rate of decay of wave processes in time and $Z$, respectively [25]. The value of $1 / \operatorname{Im} k$ sometimes defined as the interval damped wave propagation. In the extreme case, the elastic range spread endless. The degree of attenuation of wave process in the time period is characterized by the logarithmic decrement

$$
\delta_{c}=2 \pi|\operatorname{Im} \omega| / \operatorname{Re} \omega
$$

Decrement is similar to the spatial

$$
\delta_{y}=2 \pi|\operatorname{Im} k| / \operatorname{Re} k .
$$

You can also introduce the concept of phase velocity of its own and steady motions

$$
c_{c}=\frac{\operatorname{Re} \omega}{R}, c_{y}=\frac{\omega}{\operatorname{Re} k}
$$

The values $C_{c}$ and $C_{y}$ have physical sense speeds of zero state at its own and steady oscillations, respectively, and, in contrast to the elastic (real) case, do not coincide with each other at the same frequencies. Two types of oscillations (and 
set their own), you can put two different formulations of the problem. And in the non-stationary case, namely the Cauchy problem for an infinite shell and boundary value problem for the semi-infinite interval changes $Z$. In either case, the solution is using the integral transformation of the decisions of the respective steady-state problems. For example, in the case of the Cauchy problem, the main vector of unknowns $\bar{Y}^{c}$. It can be in a superposition of waves

$$
\bar{Y}^{c}=(r, z, t)=\sum_{n} \int_{-\infty}^{\infty} Y_{n}^{c}(r, k) \exp \left[t\left(k z-\varpi_{n}(k) t\right)\right] \mathrm{d} k
$$

where vectors $\bar{Y}_{n}^{c}$ are their own form of the problem of natural oscillations, normalized so that the spatial Fourier spectrum of the initial disturbance $\bar{f}(r, z)=\bar{Y}^{c}(r, z, 0)$ forms a linear combination

$$
\bar{f}(r, z)=\int_{-\infty}^{\infty} F(r, k) \mathrm{e}^{i k z} \mathrm{~d} k, \bar{f}(r, k)=\sum_{n} \bar{Y}_{n}^{c}(r, k) .
$$

Similarly, the main vector of unknowns $\bar{Y}^{y}$ boundary value problem is calculated according to the expression

$$
\bar{Y}^{y}(r, z, t)=\sum_{n} \int_{-\sigma}^{\sigma} \bar{Y}_{k}^{y}(r, \omega) \exp [i k(\omega) z-\omega t] \mathrm{d} \omega
$$

where $\bar{Y}_{k}^{y}$ forms steady-state oscillation, the linear combination of which should form a Fourier spectrum given boundary perturbation

$$
\bar{q}(r, t)=\bar{Y}^{y}(r, 0, t), \bar{q}(r, t)=\int_{-\infty}^{\infty} q(r, \omega) \mathrm{e}^{-i \omega t} \mathrm{~d} \omega, \bar{q}(r, \omega)=\sum_{n} \int_{-\infty}^{\infty} Y_{n}^{y}(r, \omega)
$$

Obviously, the solutions (8) and (9) have a meaning only when there are (10) and (11). So, there are four possible variants of steady motions, which are discussed below, and established their own systems fluctuations shell-fluid inside and outside the sheath liquid [25]. Substituting the solution (7) in the system of differential Equations (2) - (6) we obtain a system of ordinary differential equations with complex coefficients, which is solved by Godunov's orthogonal sweep method with a combination of method of Muller [26] in the complex arithmetic.

\section{Torsional Vibrations}

After performing in (5) the change of variables (7) permitting relations describing stationary torsional vibrations of the shell liquid, formulated in the form of the spectral boundary value problem for a system of two ordinary differential equations

$$
\begin{aligned}
& \frac{\mathrm{d} \tau_{\varphi}}{\mathrm{d} r}=-\left(\rho_{0}^{*} \omega^{2}-i \mu^{* 2} \xi^{2} \omega\right) \vartheta_{\theta}-\frac{2 \tau_{\varphi}}{r} \\
& \frac{\mathrm{d} v}{\mathrm{~d} r}=\frac{\vartheta_{\theta}}{r}+\frac{i}{\omega \mu^{*}} \tau_{\varphi} \\
& r=R_{1}: h_{0}\left(\bar{G} \xi^{2}-\xi \rho_{0} \omega^{2}\right) \vartheta_{\theta} \pm \tau_{\varphi}=0 \\
& r=0: \tau_{\varphi}=0
\end{aligned}
$$

First investigate fluctuations of fluid in the walls. Equations (12) can be con- 
verted to a single equation for the displacement $V$

$$
\frac{\mathrm{d}^{2} \vartheta_{\theta}}{\mathrm{d} r^{2}}+\frac{\mathrm{d} \vartheta_{\theta}}{r \mathrm{~d} r}+\left(-\xi^{2}+i \frac{\omega}{v^{*}}-\frac{1}{r^{2}}\right) \vartheta_{\theta}=0 ; \quad v^{*}=\frac{\mu^{*}}{\rho_{0}^{*}}
$$

The solution of Equation (13) is limited at $r=0$ has the form

$$
v=A_{1} J_{1}\left(r \sqrt{-k^{2}+i \frac{\omega}{v^{*}}}\right)=0 .
$$

where $J_{1}$-Bessel function of the first order, and $A$ is an arbitrary constant. Given the immobility of the shell, we obtain the dispersion equation

$$
J_{1}\left(R_{1} \sqrt{-k^{2}+i \frac{\omega}{v^{*}}}\right)=0
$$

from whence

$$
\omega_{n}=-i\left(v^{*} k^{2}+\Gamma_{m}^{2}\right)
$$

in the case of natural oscillations and

$$
k_{n}=\sqrt{-\Gamma_{n}^{2}+i \frac{\omega}{v^{*}}}
$$

in the case of steady-state oscillations. Here, through the $\Gamma_{n}$ marked the roots of Bessel functions assigned to $R$. As it can be seen from (15), (16) own motion aperiodicity always on time, with the anchor points are fixed (the phase velocity $C_{0}=0$ ), while the steady motion are oscillatory in nature, as the nodal point move at the speed of $C_{p}$, a monotonically increasing from zero to indefinitely with an increase in viscosity or $v^{*}$. These characteristic features of the motion of a viscous medium will appear in the following more complex example.

Let us now consider the relation (12) in the case of the internal arrangement of the liquid. This problem can be solved in the same way using special features and have a dispersion equation

$$
-k^{2}+\frac{\omega^{2}}{a^{2}}+\frac{\omega v^{*}}{a^{3} \tilde{p} \tilde{h} R^{2}}+\left(z \frac{J_{0}(z)}{J_{1}(z)}-2\right)=0
$$

which was first obtained in $A$. Guz [22]. Here we have introduced new designations

$$
\tilde{p}=\frac{\rho^{*}}{\rho_{0}} ; \tilde{h}=\frac{h}{R_{1}} ; z=R_{1} \sqrt{-k^{2}+i \frac{\omega}{v^{*}}} ; a=\sqrt{\frac{G}{\rho_{0}}}
$$

shear wave velocity shell: $J_{0}$-Bessel function of zero order.

The direct solution of the Equation (18) comes up against certain difficulties caused by the need to calculate the Bessel functions of complex argument. Therefore we examine (18) by means of asymptotic representations of these functions at small and large arguments $z$. The smallness of $z$ occurs in the low-frequency vibrations. According to the known expansion $J_{0}$ and $J_{1}$ power series

$$
J_{0}=1-\frac{z^{2}}{4}=\cdots ; J_{1}(z)=\frac{z}{2}\left(1-\frac{z^{2}}{8}+\cdots\right) ;
$$


Hold the expansions (19) only the first term, we obtain

$$
-k^{2}+\frac{\omega}{a^{2}}=0
$$

dispersion equation of torsional vibrations or dry shell filled with an ideal liquid, keeping in (19) on the first two terms, we have the equation

$$
-k^{2}+\frac{\omega^{2}}{a^{2}}+i \frac{\omega v^{*}}{4 a^{2} \tilde{p} \tilde{h}}\left(k^{2}-i \frac{\omega}{v^{*}}\right)=0
$$

the root of which, for example, in the case of steady-state oscillations is given by

$$
k=\frac{\omega}{a}\left[\left(1+\frac{1}{4 \tilde{p} \tilde{h}}\right) /\left(1-\frac{\omega v^{*}}{4 a^{2} \tilde{p} \tilde{h}}\right)\right]^{1 / 2} .
$$

The physical interpretation of (18) is provided below. Consider now the situation when $z$ is large enough, which corresponds to a high-frequency vibrations and low viscosity. In this case the asymptotic formulas for the Bessel functions have the form

$$
J_{0}(z) \cong\left(\frac{2}{\pi z}\right)^{1 / 2} \cos \left(z-\frac{\pi}{4}\right), J_{1}(z) \cong\left(\frac{2}{\pi z}\right)^{1 / 2} \sin \left(z-\frac{\pi}{4}\right)
$$

On the basis of (20) and (21) it is easy to show that for sufficiently large positive imaginary part $z$ : $J_{0}(z) / J_{1}(z) \cong-i$. Substituting (1) and further assuming smallness $v^{*}$ in comparison with the $\frac{\omega}{k^{2}}$, to obtain an approximate dispersion equation, which is also contained in the [17]

$$
-k^{2}+\frac{\omega^{2}}{a^{3}}\left(1+\sqrt{\frac{v^{*}}{\omega}} \frac{\tilde{p}}{\tilde{h} R} \frac{l+i}{1.41}\right)=0
$$

where, in the pursuit of the viscosity $v^{*}$ to zero (and also tends $\omega$ to infinity), we have a trivial result $\frac{\omega}{k} \rightarrow 0$, which was obtained at low $\omega$ from Equation (20). Equation (22) when an unacceptably high viscosities. In this case, the phase velocity $C$ unlimited increases with $\omega$. This example shows inconsistencies of various asymptotic estimates in the mid-frequency vibrations. Thus, the analysis of wave processes asymptotic methods in the first approximation is not possible to establish the limits of applicability of formulas and calculations to estimate the error. In this paper for solving spectral problems using a direct numerical integration of permitting relations of the type (12) by the method of orthogonal shooting in complex arithmetic. This approach avoids the above difficulties associated with the calculation of Bessel functions of complex argument. Another advantage is due to the specificity of the orthogonal sweep method, which is due to the procedure orthonormality can solve highly rigid system with a boundary layer. As a result of a numerical study has found that the problem of natural oscillations (12) admits no more than one complex value $\omega$, corresponding vibrations of the shell together with the adjacent liquid layers to it. The rest found the Eigen values appeared purely imaginary. They correspond to a periodic motion 
of a fluid with almost stationary shell. Proper form corresponding complex values also are complex, that is, the phase of joint oscillations of the shell and liquid is not the same along the radius. In the case of steady-state oscillations all the calculated Eigen values $k$ and their own forms are complex.

\section{Numerical Results}

Consider the case of natural oscillations, when the shell is filled with liquid. In Figures 1-9 and Figure 1, Figure 2, Figure 4, Figure 5 show, respectively, depending on the dispersion curves $\operatorname{Re} \omega, \operatorname{Im} \omega, \delta$ the wave number, $k$-the first mode, in which the damping coefficients of the smallest, and the Eigen values are complex Bat. In accordance with the numbering of graphs asked four different values of the coefficient $\eta$ 1) $0.0009: 2) 0.0018$ 3) 0.15 4) 0.018 ( $a=0.6199$; $\tilde{\rho}=0.0529 ; \tilde{h}=0.0101 ; R=1 ; v_{0}=0.25$.) for the remaining parameters according to (1) In Figure 3, Figure 6, Figures 7-9 to show their own forms Rev for values $k$ equal to 1 and 8 , respectively. It is easy to notice the difference in the behavior characteristic of the dispersion curves 1.2 and 3.4. In the last two cases, there is a wave number since a variable with only takes purely imaginary values corresponding to a periodic motion of the system. For curves 1.2 with less viscosity real part of the Eigen values $\operatorname{Re} \omega$ nonzero at all wave numbers and the damping rate has a finite limit at infinity. The greater the viscosity, the earlier start a periodic traffic (curves 3,4 ) and the higher limit of the damping rate

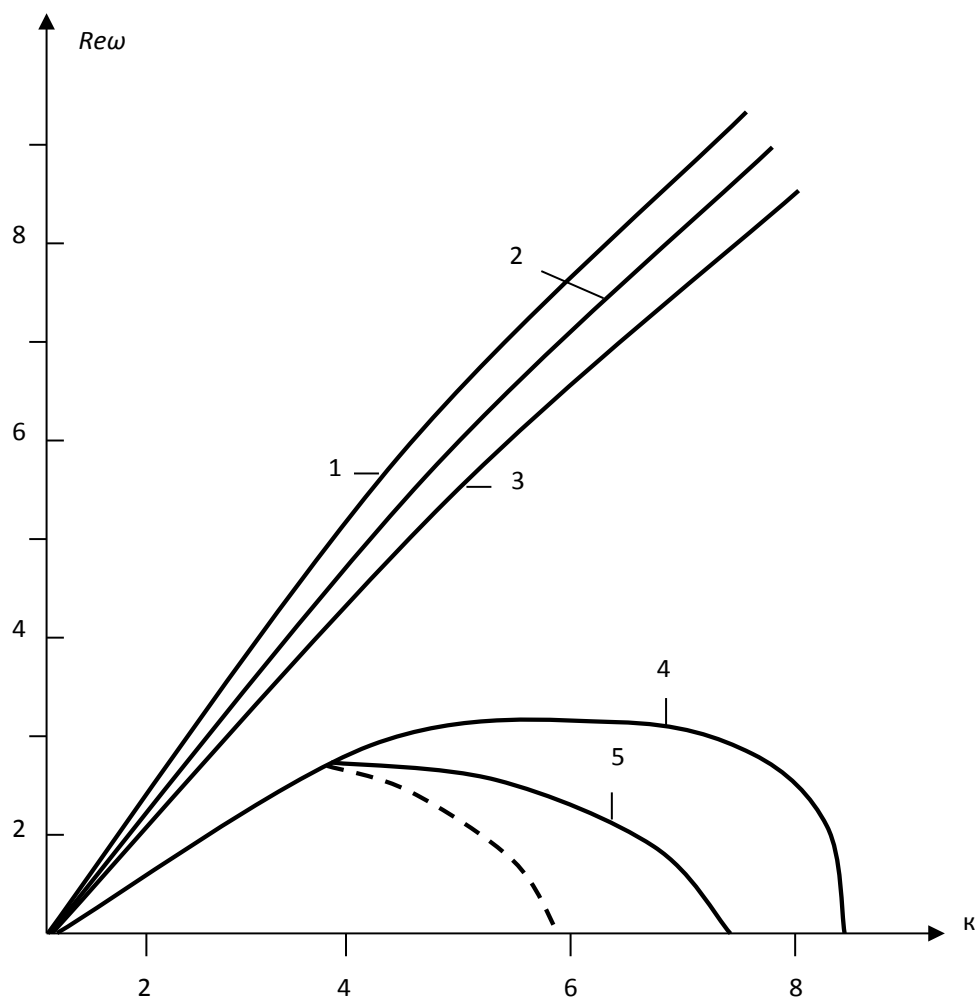

Figure 1. Dependence of the real part of the complex frequencies $(\operatorname{Re} \omega)$ to wave numbers $(k)$ for different values of $\eta$. 1-0.0009; 2-0.0018; 3-0.18, 4-0.19, 5 -according to the formula (20); By the formula (22). 


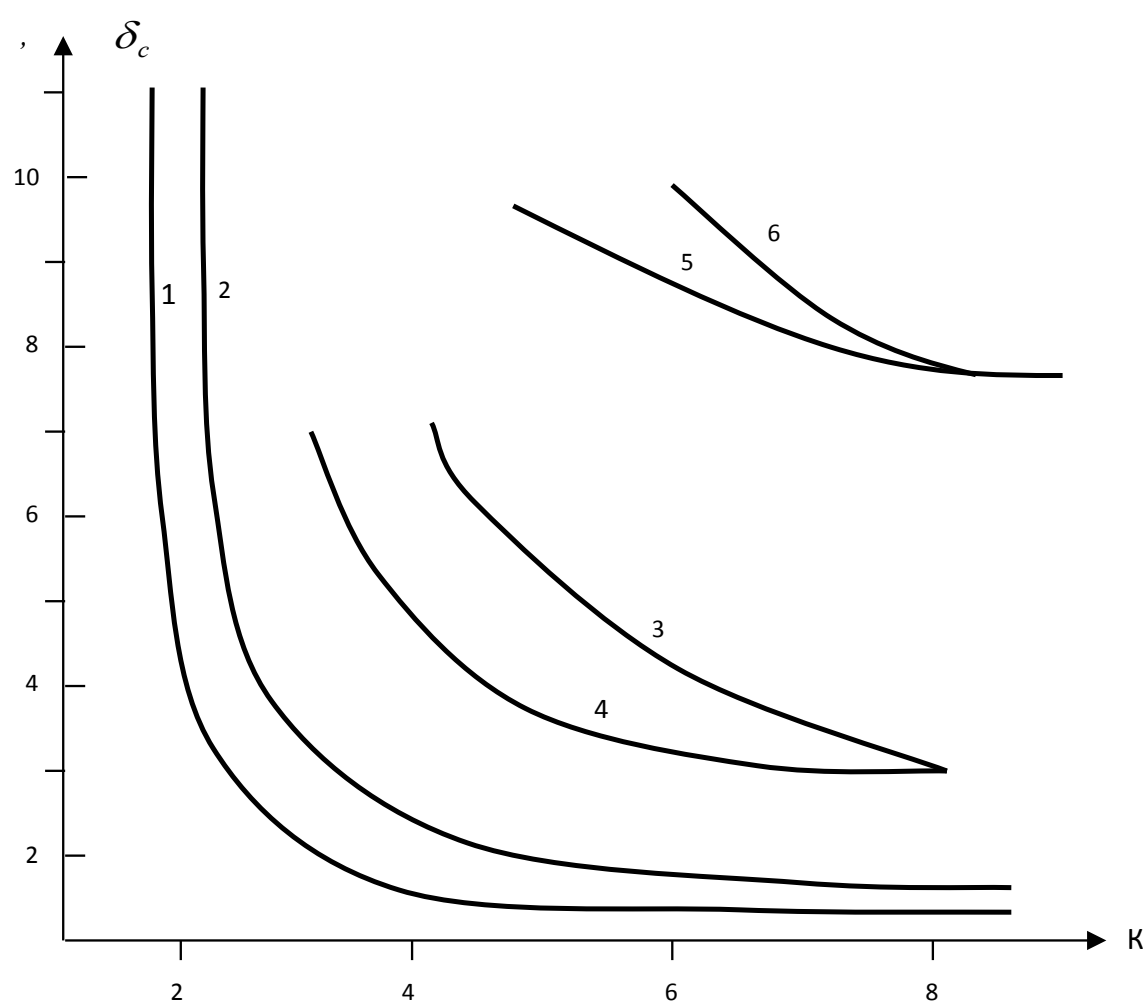

Figure 2. Dependence of the logarithmic decrement $\left(\delta_{c}\right)$ on the wave numbers $(k)$ for different values of $\eta$. 1-0.0009; 2-0.0018; 3-0.18, 4-0.19, 5-0.20; 6-0.22.

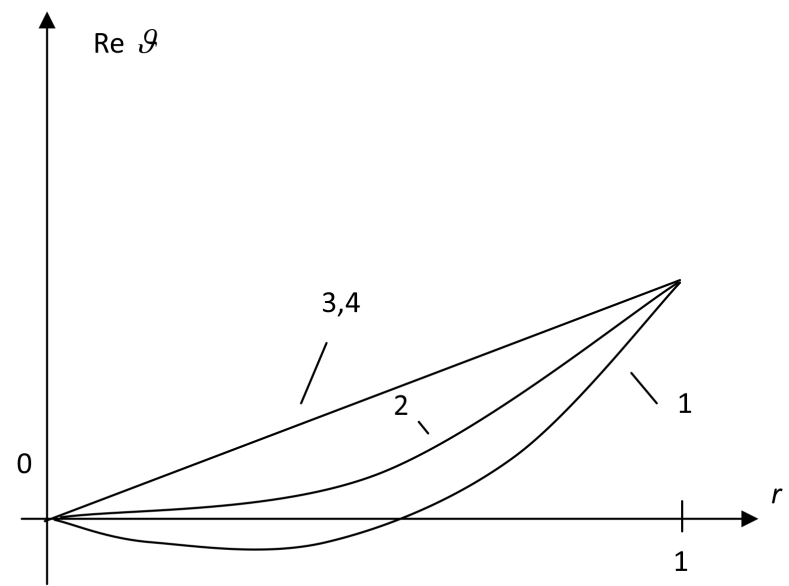

Figure 3. Dependence of $\mathcal{\vartheta}$ on the wave number $r$, for different values of the viscosity of a liquid. 1-0.0009; 2-0.0018; 3-0.18; 4-0.19.

(curves 1,2 ). It follows that where is a minimum critical viscosity $\eta_{k}$ above which a zone of high wave numbers of the first mode, there are a periodic wave number. As a result of numerical experiment, it was found that the critical values of the coefficient of viscosity $\eta_{k}$ is in the range [0.0120,0.0125]. Analyzing the dependence of energy dissipation on the wave number, two opposite tendencies should be noted. As the wave number increases, at a fixed amplitude, tangential 


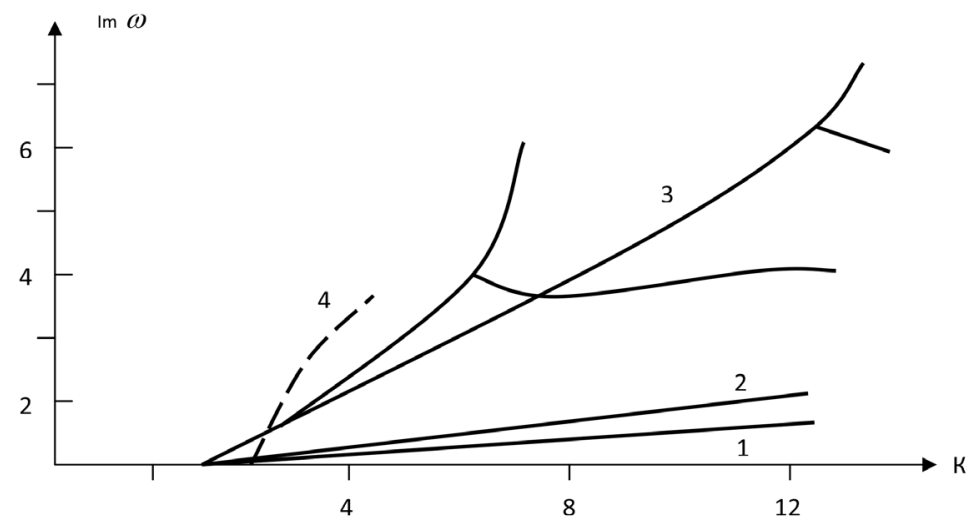

Figure 4. Dependence of the imaginary part of the complex frequencies (Im) to wave numbers $(k)$ for different values of $\eta: 1-0.0009 ; 2-0.0018 ; 3-0.18$; 4-0.19; by the formula (22).

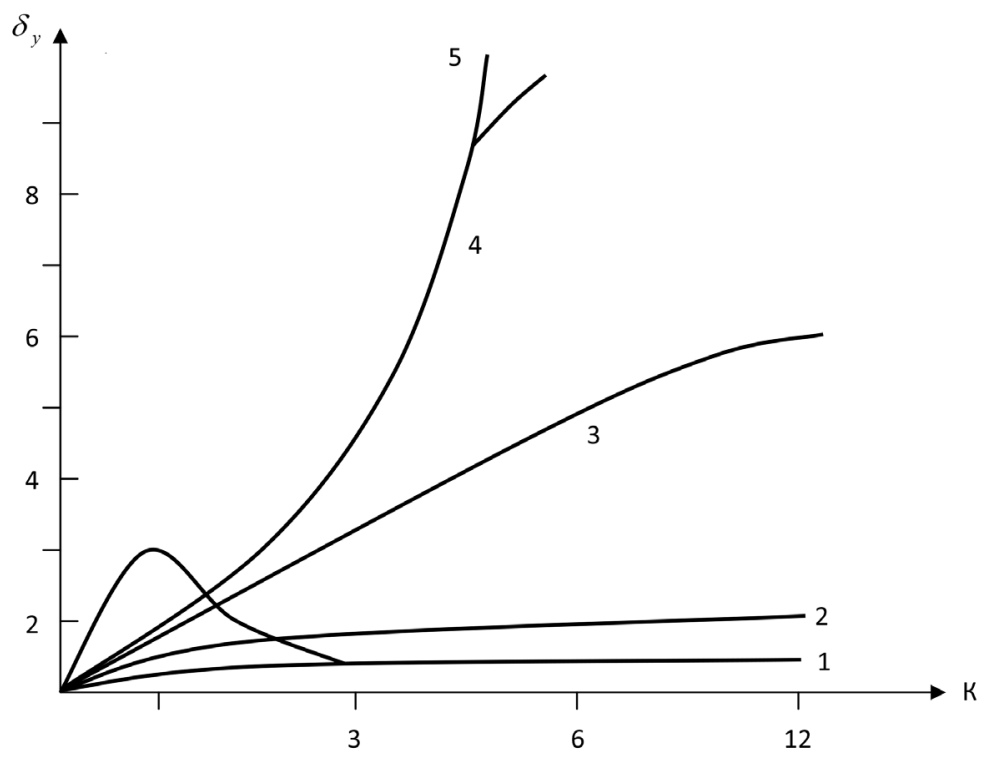

Figure 5. Dependence of the spatial decrement on the wave number $k$ for different values of $\eta$ : 1-0.0009; 2-0.0018; 3-0.18; 4-0.19.

stresses linearly increase according to (6): c another, as shown in Figure 3, localization of the fluid motion amplitudes near the shell simultaneously results, which leads to a decrease in the mass of fluid involved in the motion, as well as tangential stresses. The difference in the behavior of curves 1, 2 and 3, 4 is due to which of the two tendencies prevails. At small wave numbers, a linear dependence of the Eigen function $v$ on the radius is observed, that is, the entire mass of the liquid is involved in the motion. Ask increases, the central part of the liquid begins to "not keep up" with the vibrations of the shell, which leads to the localization of the amplitudes. The rate of localization depends on the viscosity of the liquid. If the localization occurs slowly, then starting from some $k$ (owing to the growth of stresses), the self-motions become aperiodic (curves 3,4). If, on the other hand, the average amplitude of the fluid oscillation decreases rapidly 


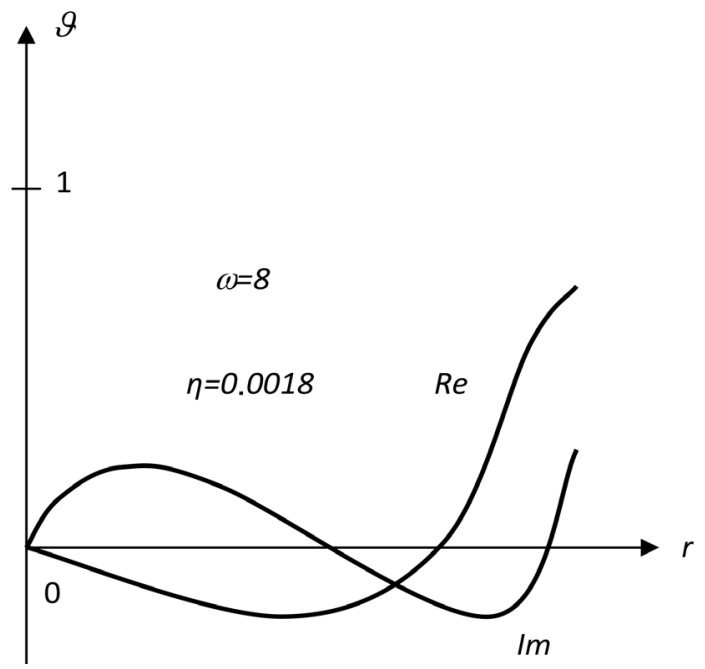

Figure 6. Dependence of $\vartheta$ on the wave number $r$. When $\omega=8, \eta=0.0018$.

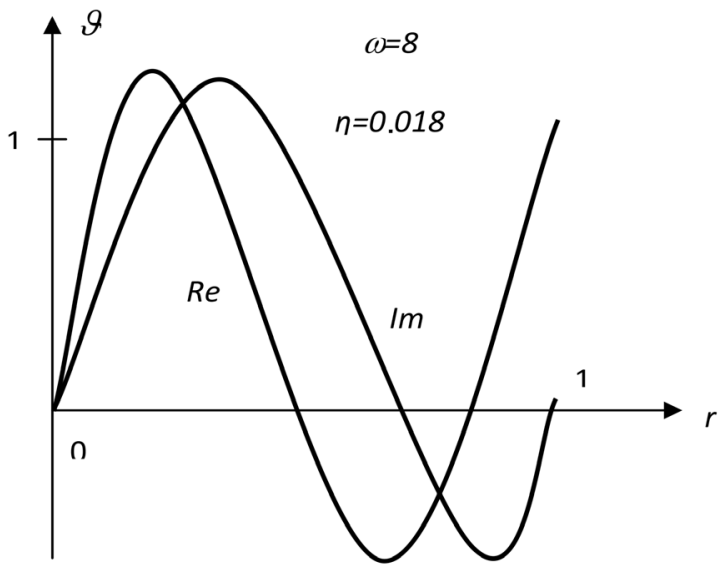

Figure 7. Dependence of $\mathcal{\vartheta}$ on the wave number. When $\omega=8, \eta=0.018$.

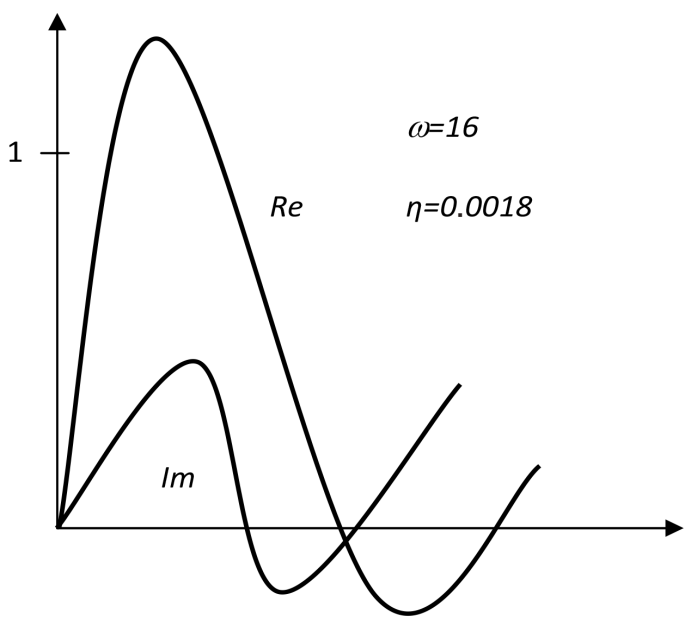

Figure 8. Dependence of $\mathcal{\vartheta}$ on the wave number $r$. When $\omega=16, \eta=0.0018$. 


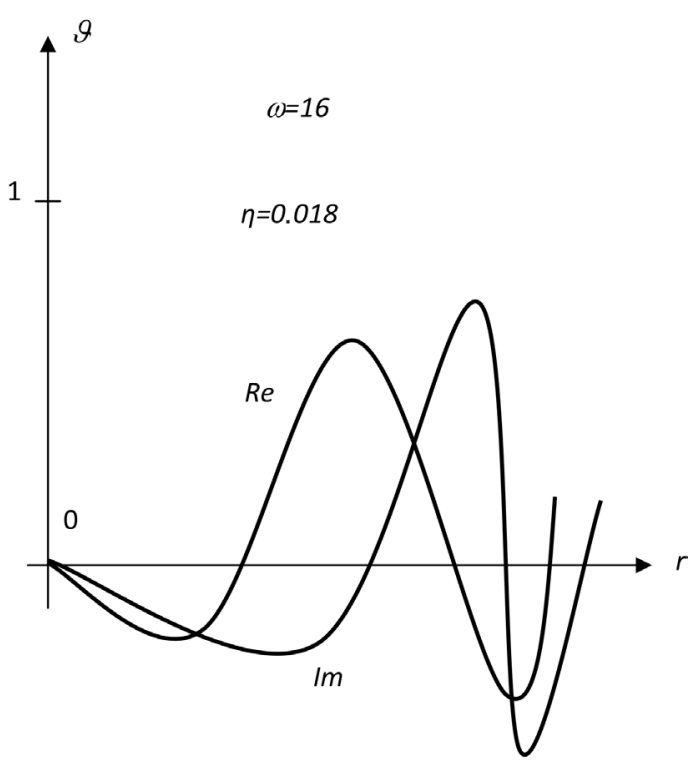

Figure 9. Dependence of $\mathcal{\vartheta}$ on the wave number $r$. When $\omega=16, \eta=0.018$.

enough, the motions will always remain oscillatory (curves 1,2 ). In this case, large voltage wave numbers prevail over voltages, and increase with increasing localization. In view of the latter circumstance, the damping coefficient always increases with increasing $k$. The linear dependence of the shape on the radius at small $\mathrm{k}$ also indicates the fulfillment of the flat-section hypothesis on which the elementary theory of viscoelastic rods is based. Using the Ritz method one can find the parameters of the Fought core model and determine the limits of applicability of this model in the framework of the hydrodynamic theory, but for a narrower class of straight rods of circular cross section. Variation equation of the principle of possible displacements, equivalent to the relations

$$
\begin{aligned}
& \int_{v} h\left(\frac{\partial u_{\varphi}}{\partial z} \delta \frac{\partial u_{\varphi}}{\partial z}+\rho_{1} \frac{\partial u_{\varphi}}{\partial z} \delta u_{\varphi}\right) R_{1} \mathrm{~d} \varphi \mathrm{d} z \\
& -\int_{v}\left(\sigma_{r \varphi} \delta \varepsilon_{r \varphi}+\sigma_{z \varphi} \delta \varepsilon_{z \varphi}+\rho_{0} \frac{\partial^{2} u_{\varphi}}{\partial z^{2}} \delta u_{\varphi}\right) r \mathrm{~d} \varphi \mathrm{d} r \mathrm{~d} z=0
\end{aligned}
$$

has the form. Choosing a linear function as the basis

$$
u_{\varphi}(r, z, t)=\varphi(z, t) r,
$$

and after substituting (24) into (23) and the standard procedure, we obtain where the parameters $\beta$ and $a_{0}$ are expressed in terms of the polar moments of inertia of the shell $I_{1}$ and liquid $I_{0}$ as follows

$$
\beta=\frac{\eta I_{0}}{G I_{1}} ; a_{0}=a /\left(1+\frac{I_{0}}{\tilde{\rho} I_{1}}\right)^{\frac{1}{2}}
$$

Equation (23) describes the torsional vibrations of a viscoelastic Feucht rod according to the relations 


$$
\left(1+\beta \frac{\partial}{\partial t}\right) \frac{\partial^{2} \varphi}{\partial z^{2}}=\frac{1}{a_{0}^{2}} \frac{\partial^{2} \varphi}{\partial t^{2}}
$$

The solution of (25) is represented in the form

$$
\varphi(z, t)=\varphi_{0} \exp (i(k z-\omega t)) .
$$

where the following relations satisfy

$$
a_{0}^{2} \kappa^{2}(1-i \omega \beta)-\omega^{2}=0 .
$$

Taking into account the relation $I_{1} / I_{0}=4 h$, it is easy to see that Equation (26) coincides with Equation (22), which was obtained for the asymptotic solution of problem (16) for small oscillation frequencies. In Figure 1, Figure 4, the dotted lines show the dispersion curves of natural oscillations found from Equation (22). As follows from the figures, a satisfactory coincidence of dotted and continuous lines is observed in the region of small wave numbers whose upper bound exceeds unity in this case and increases with increasing viscosity of the liquid. In the short-wavelength range, there is a discrepancy due to the localization of the oscillation amplitudes near the shell. Small wave numbers correspond to the natural vibrations of long finite tubes. We now turn to an analysis of the steady-state oscillations of a shell filled with a liquid. Figures 1-9 shows the dispersion curves and waveforms for two values of the viscosity coefficient (below and above the critical value) 1) $0.0018,2) 0.018$ and the same values of the remaining parameters as in (22). In the first case of relatively low viscosity, the results of the calculation are in good agreement with the asymptotic solutions of the Goose Equation (18) at high frequencies.

\section{Longitudinal-Transverse Vibrations}

This section analyzes the stationary longitudinal-transverse vibrations of a shell filled with fluid, which according to (6) can be described by a system of four ordinary differential equations

$$
\begin{aligned}
\frac{\mathrm{d} \vartheta_{r}}{\mathrm{~d} r} & =-\frac{\vartheta_{r}}{r}-i k \vartheta_{z}-p \\
\frac{\mathrm{d} \vartheta_{z}}{\mathrm{~d} r} & =i k \vartheta_{r}+\frac{1}{\eta \omega} \tau_{\check{y}} \\
\frac{\mathrm{d} \sigma_{r}}{\mathrm{~d} r} & =-\rho_{0} \omega^{2} \vartheta_{z}+2 i \eta \omega\left(\frac{\mathrm{d} \vartheta_{r}}{\mathrm{~d} r}-\frac{\vartheta_{r}}{r}\right)=i k \tau_{z} \\
\frac{\mathrm{d} \tau_{z}}{\mathrm{~d} r} & =-\rho_{0} \omega^{2} \vartheta_{z}+2 \eta \omega k\left(\frac{\mathrm{d} \vartheta_{r}}{\mathrm{~d} r}-i k \vartheta_{z}\right)-i k \sigma_{r}-\frac{\tau_{z}}{r}
\end{aligned}
$$

With the boundary conditions

$$
\begin{aligned}
& r=0: \vartheta_{r}=0, \tau_{z}=0 ; \\
& r=R: D \nabla^{4} u+\frac{C}{R}\left(\frac{u}{R}+i v_{0} k w\right)+\sigma_{r}-\rho_{1} h \omega^{2} u=0 ; \\
& C\left(i v_{0} k \frac{u}{R}-\nabla^{2} u\right)-\tau_{z}+\rho_{1} h \omega^{2} w=0 ; C=\frac{E h_{0}}{1-v_{0}^{2}} .
\end{aligned}
$$


The value of $p$ in the first equation of system (27) is defined through the main unknowns according to the expression

$$
p=\frac{-\sigma_{r}+2 i \eta \omega\left(i k u+\frac{\vartheta_{r}}{r}\right)}{\rho_{0} C_{0}^{2}-i \omega(k+2 \eta)}
$$

The spectral problems (27), (28), as in the case of longitudinal-transverse vibrations were solved by orthogonal shooting. To find the roots of the characteristic equation method were used Mueller.

\section{Numerical Results}

The results of numerical study of natural oscillations. Figure 10 shows the dispersion curves $\operatorname{Re} \omega$ the wave number $k$ for the case of an incompressible $\left(C_{0}=\infty\right.$ - dot-dash line) and compressible ( $C_{0}=0.1$ - solid line) of the liquid. Shell parameters and coefficients of viscosity taken following: $h_{0}=0.05 ; p=1.8 ; v_{0}=0.25$; $h=6.011 \times 10^{-4} ; K=-2 \eta / 3$. Here and henceforth given dimensionless quantities for which the units of length and mass density are $R, R\left(\frac{\rho_{0}}{E}\right)^{\frac{1}{2}}, \frac{1}{\rho_{0}}$. For an incompressible fluid, there are two modes, corresponding mainly longitudinal (curve 1) and preferably a cross (curve 2) fluctuations in the shell, with complex Eigen values. All other traffic have their own imaginary Eigen values, that is a periodic in time. The dashed lines in Figure 11 are designated the dispersion curves corresponding to the vibrations of a shell with an ideal incompressible fluid. The solution of the latter problem is given below. It should be noted that, unlike the dry shell joint oscillations transverse vibrations of said sheath fluid density $p_{1}$, it takes place on a smaller compared to the frequency of longitudinal vibrations in the entire range of the wave number. When administered viscosity oscillation frequency of the first mode decreases, apparently due to the involvement of additional masses in movement of fluid in the boundary layer and in the second mode appears critical wave number restricting oscillatory motions bottom region. In [25], who investigated the steady oscillations, noted the desire for zero phase velocity of the lowest mode with decreasing frequency. Proper motion of the shell and the viscous compressible fluid has an infinite number of modes. The paper S. Vasin et al. [26] using asymptotic methods of solving, the latter effect could not be found. Figure 11 shows the dispersion curves for the first four events with a minimum of vibration frequencies (curves $3,4,5,6$ ) in ascending order of magnitude Re $\omega$. Comparing curves 1.2 and 3.4 together, we can see that the second worse than the first few vibration modes of the shell-compressible fluid to the selected parameters are satisfactorily described by a model of an incompressible fluid in the region of wave numbers $k<1$. This gives grounds for the study of the said system in the first approximation neglect the compressibility of the fluid. System elastic shell is a viscous liquid dissipations-inhomogeneous viscoelastic body at a radial coordinate. Moreover, in contrast to the earlier torsional vibrations here for an incompressible fluid, there are 


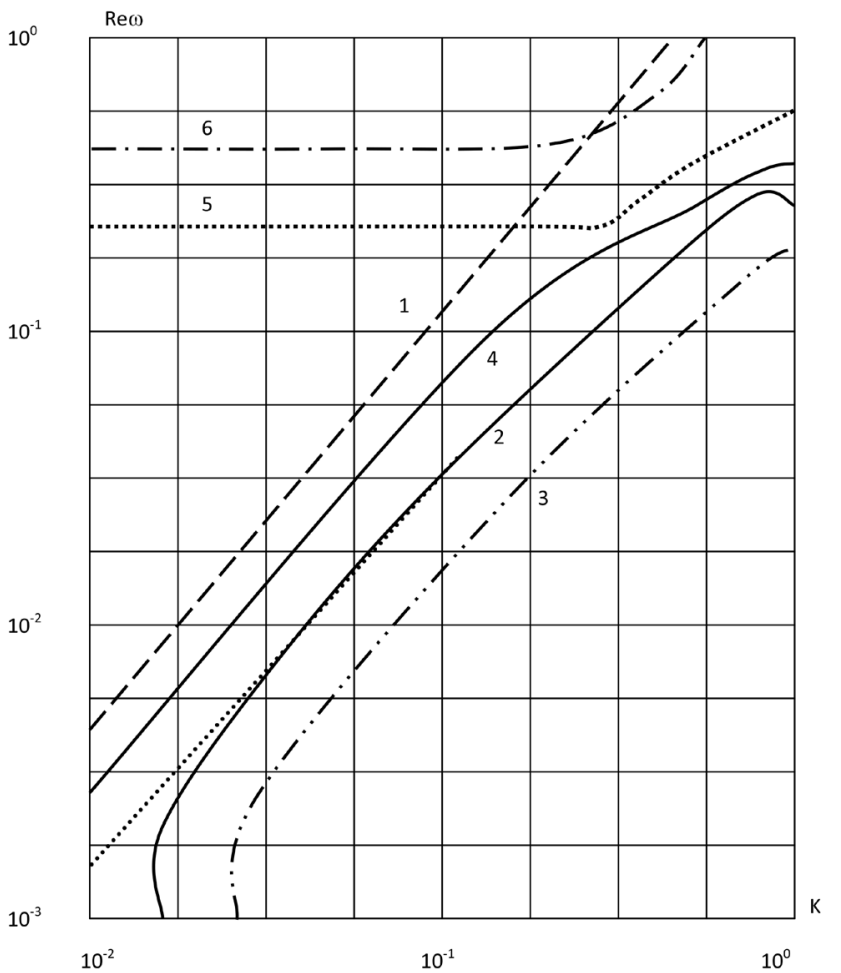

Figure 10. Addiction $\operatorname{Re} \omega$ with the wave number $k$ in the case of an incompressible fluid.

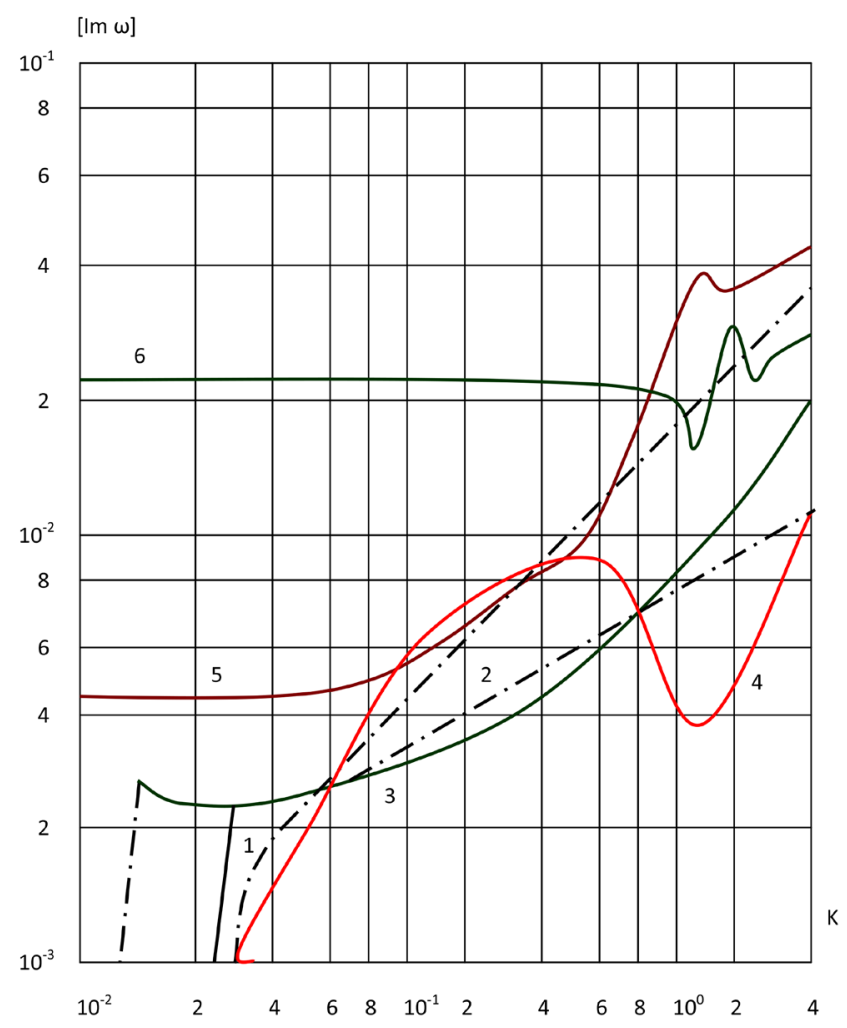

Figure 11. Addiction Im a the wave number in the case of a compressible fluid. 
two, and compressible-unlimited number of vibration modes. It is interesting to find out how this system can be shown a synergistic effect. Figure 11 shows the dispersion curves (2) for the following parameters of the shell and liquid:

$$
h=0.05 ; \rho_{3}=80 ; v=0.25 t ; \eta=7.071 \times 10^{-4} ; C_{0}=\infty
$$

Dash-dotted lines correspond to fluctuations in the dry shell. The dashed lines show the frequency dependence for the case of an ideal fluid $v=0$. In contrast with the previously discussed embodiment, the density $p=8$, in this case partial frequency $(v=0)$ of the longitudinal and transverse vibrations of the shell with a perfect fluid intersect. It is natural to expect that the $v$ near the intersection of partial frequencies will be a strong connectedness of both modes, leading to increased energy, resulting in a synergistic effect. Indeed, the presence of events demonstrates the effect of the conversion of Vina-longitudinal mode in transverse and longitudinal cross-section in a change of the wave number in the vicinity of the intersection of partial frequencies. Violation of the monotony of growth and synergies. Compared to the previous description of this effect there are two features. Firstly, the effect is far from the place of approximation curves of two modes, secondly, damping factor curves do not intersect. In [27] investigated the coherence of joint oscillations of ideal compressible gas and the shell with the help of diagrams wines. As he examined the frequency of partial oscillations of gas in rigid walls and an empty shell. Returning to Figure 12, Figure 13 we note a similar manifestation of the effect of wines in places of convergence curves 4.5 and 5.6. In these areas in Figure 14 there is a synergistic effect for the curves. It is interesting to trace the influence of fluid viscosity on connectivity modes. 3.4 Curves in Figure 15 correspond to the value of the viscosity coefficient $\eta=0.11$ at constant other parameters. In this case, fashion predominantly transverse vibrations are defined on a finite interval of the wave of change, and the effect of guilt is not observed, indicating a loose coupling modes. Another large increase in viscosity ( $\eta=0.13$, curve 5 ) leads to the fact that fashion is everywhere transverse vibrations becomes a periodic and $y$ longitudinal oscillations appear critical wave numbers, limiting the scope of the vibration motions of the top. The physical nature of the observed effect is revealed when analyzing the vibrations of a shell filled with a perfect fluid. The equations of harmonic oscillations of an ideal liquid is easy to deduce from (27), formally putting viscosity coefficients equal to zero.

$$
\frac{\mathrm{d} \vartheta_{r}}{\mathrm{~d} r}=-\frac{\vartheta_{r}}{r}-i k \vartheta_{z}-\frac{\sigma}{\rho_{0} C_{0}^{2}}, \frac{\mathrm{d} \sigma}{\mathrm{d} r}=-\rho_{0} \omega^{2} \vartheta_{r}, \sigma=i \rho_{0} \omega^{2} \vartheta_{z}
$$

General solution of (26) satisfying the finiteness condition unknown at zero, has the form

$$
\begin{aligned}
& \vartheta_{z}=A J_{0}(q r) ; \sigma=i \rho_{0} \frac{\omega^{2}}{k} A I_{0}(q r) \\
& \vartheta_{z}=i \frac{q}{R} A I_{1}(q r) ; q^{2}=\frac{\omega^{2}}{C_{0}^{2}}-k_{0}^{2}
\end{aligned}
$$




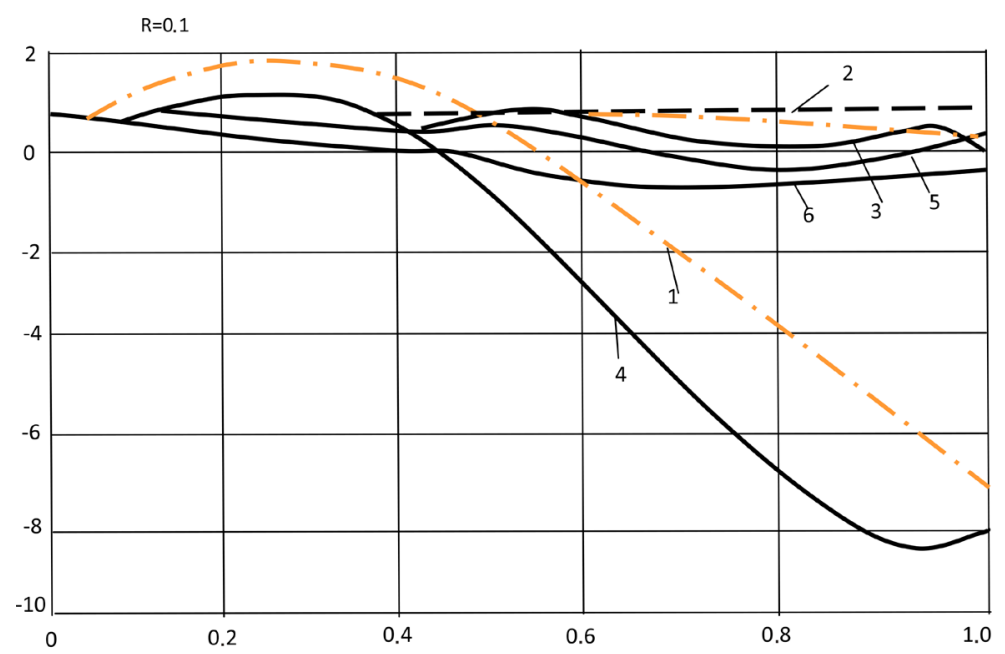

Figure 12. The dependence of $R e W$ on the wave number $r$.

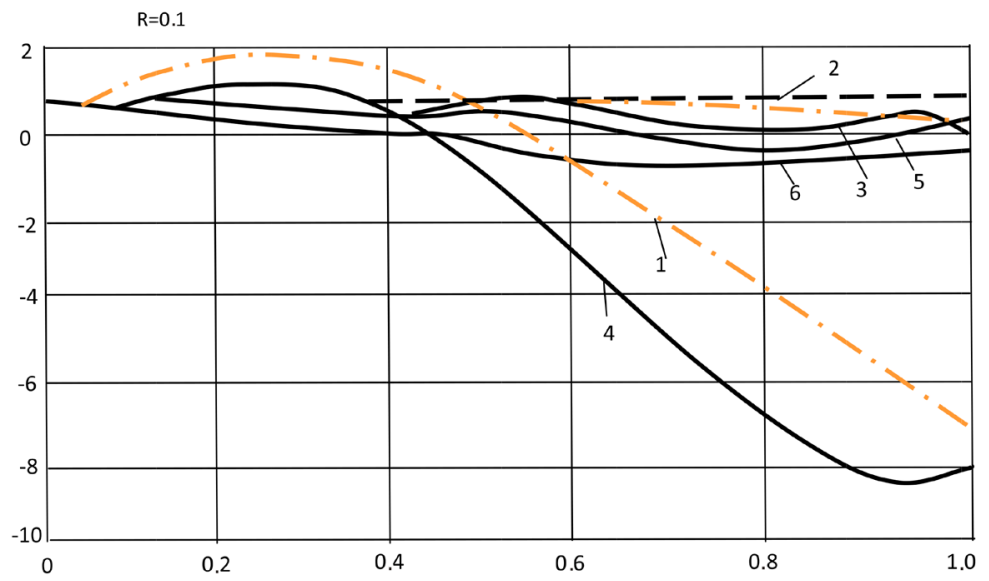

Figure 13. The dependence of $\operatorname{Re} W$ on the wave number $r$.

where $A$ arbitrary constant: $J_{0}, J_{D}$-Bessel functions of zero and first order, respectively. The boundary conditions at the $r=R$ similarly written conditions (28)

$$
\begin{aligned}
& D \nabla^{4} u+\frac{C}{R}\left(\frac{u}{R}+i v_{0} k w\right)+\sigma_{r}-\rho_{1} h \omega^{2} u=0 ; \\
& C\left(i v_{0} k \frac{u}{R}-\nabla^{2} u\right)+\rho_{1} h \omega^{2} w=0
\end{aligned}
$$

where $w$-axial movement of the shell, which is not now coincides with the axial movement of the liquid. After substitution of the solutions (22) of (23) there is a system of homogeneous linear algebraic equations in the unknown $A$ and $U_{1}$. The roots of the determinant of this system are the desired Eigen values, and its decision to define the relation between $A$ and $U_{l}$.

For an incompressible fluid, there are two real own Bessel functions $I_{0}$ and $I_{1}$

$$
\omega_{1}=R\left(\frac{E}{\rho_{1}}\right)^{\frac{1}{2}} ; \omega^{2}=\left[\frac{E}{R_{1} \rho_{1}}\left(1+h^{2} R_{1} \kappa^{4}\right) /\left(1+\frac{\rho_{n} I\left(k R_{1}\right)}{h \rho_{1} I_{1}\left(k R_{1}\right) \kappa}\right)\right]^{\frac{1}{2}}
$$




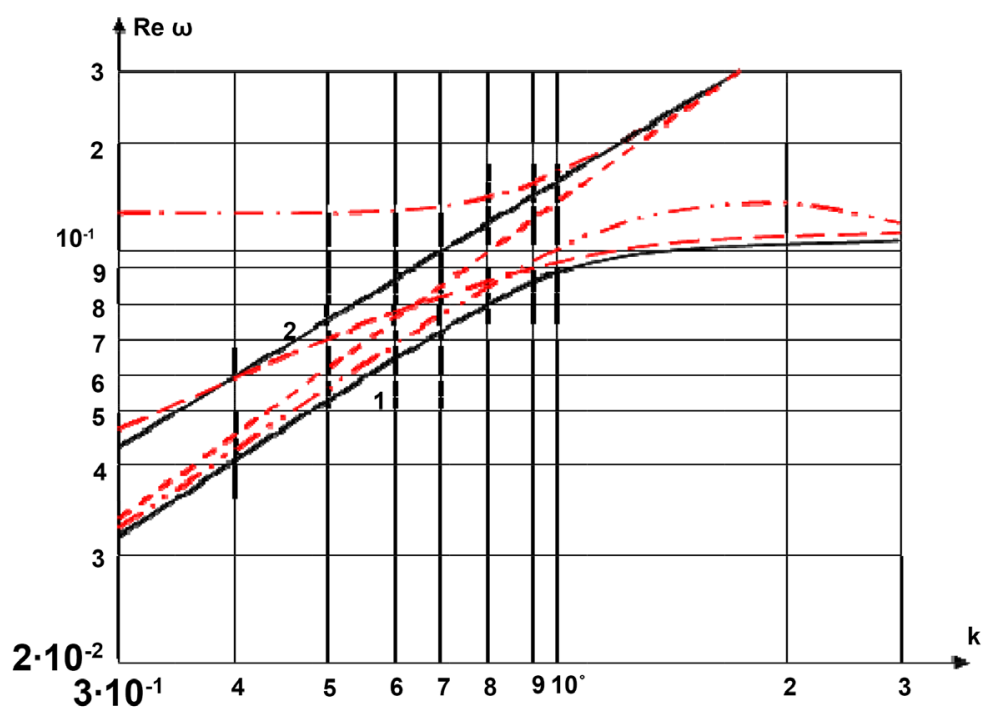

Figure 14. The dependence Rew on the wave number $k$ (for the case of an incompressible fluid).

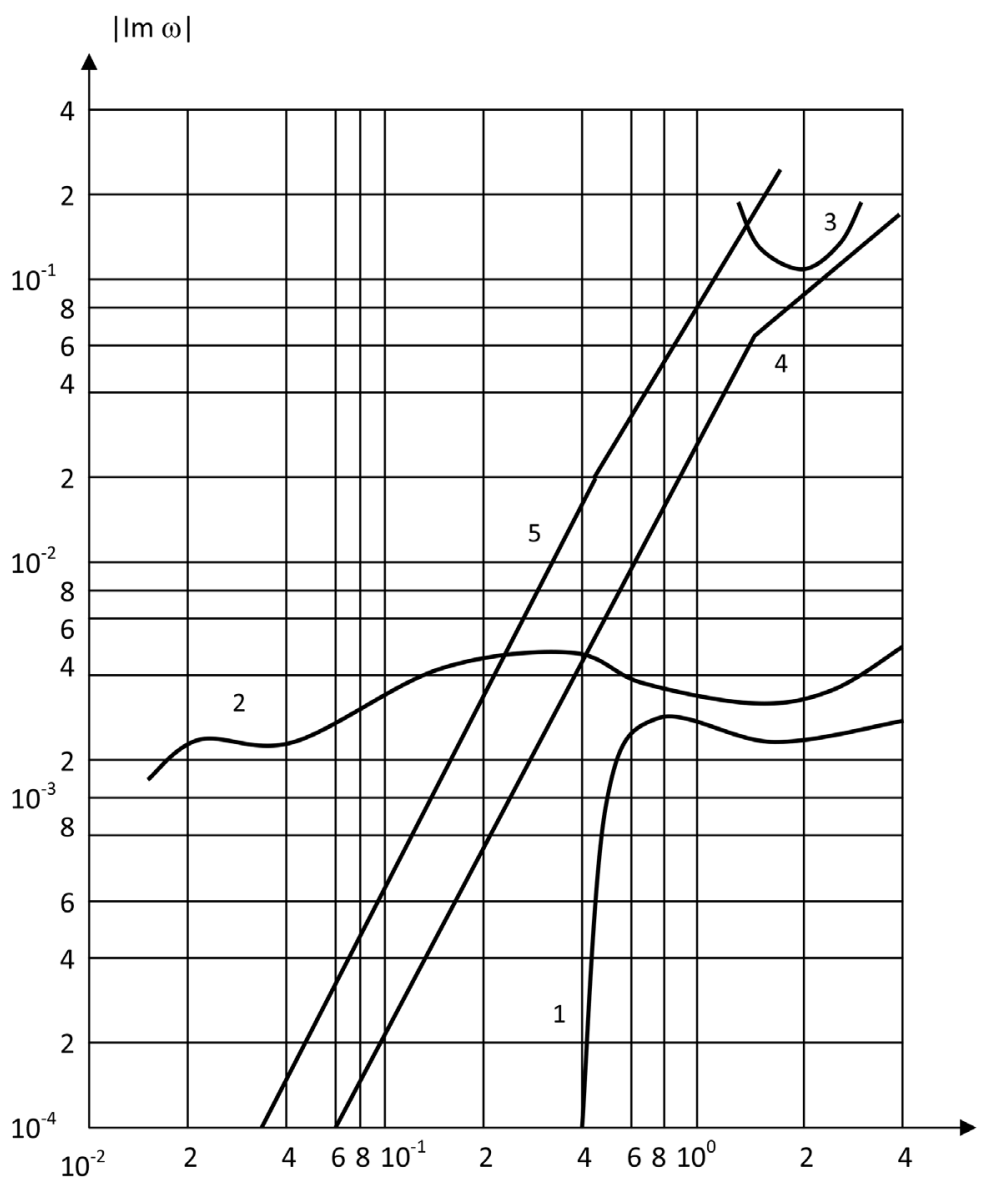

Figure 15. The dependence of $\operatorname{Im} \omega$ on the wave number $k$.

Unlike dry shell here second frequency locking is absent and the phase speed at low $k$ equal to the 


$$
C_{R}=\left(\frac{E h}{2 \rho_{0} R_{1}}\right)^{\frac{1}{2}}
$$

which coincides with the speed of the wave Ressala (see. the review at the beginning of this chapter). In the case of a compressible fluid $v=0$ and limiting the phase velocity of the transverse mode oscillation in the shell $k \rightarrow 0$ is the velocity of waves Cortège Zhukov sky.

$$
C_{k}=\frac{C_{0} C_{R}}{\left(C_{0}^{2}+C_{R}^{2}\right)^{\frac{1}{2}}}
$$

Numerical study showed that the critical value $C_{k}$ does not depend on the viscosity of the liquid, but with increasing $\eta$ weakening the dependence of oscillations of Poisson's ratio, so that the ratio $(\max i m \varpi) /(\min i m \varpi) \rightarrow 1$ and own form $U$ it becomes flat. As follows from the above results, generally within the engineering problem statement, we cannot adequately describe the longitudinal vibrations of the cylindrical shell filled with a viscous fluid via rod theory.

\section{Conclusions}

1) Numerical investigation has shown that the critical value Vk does not depend on the viscosity of the liquid, but as the value of $\eta$ increases, the dependence of the Poisson's coefficient is weakened, so that the ratio $(\max i m \omega) /(\min i m \omega) \rightarrow 1$ and the proper form $U$ becomes flat. However, in some particular cases, namely: for a large viscosity or at a critical value of the Poisson ratio, it is possible to propose a method for estimating the natural frequency based on a bar model of the type (26).

2) Analyzing the dependence of energy dissipation on the number of waves, it should be noted that there are two opposite tendencies, since the number of waves and tangential stresses $p_{z \varphi}$ increases for a fixed amplitude $v$. And also, as shown by numerical results, localization of fluid motion amplitudes near the shell occurs simultaneously, which results in a decrease in the mass of fluid involved in the motion, as well as tangential stresses $p_{r \varphi}$.

3) For low viscosity, the frequency Rek of both modes is close to one in the low-frequency region, and at high frequencies the phase velocity $\mathrm{C}_{\mathrm{y}}$ corresponds to the tendency to speed in the dry shell. Damping coefficients grow approximately linearly, and in the second mode, this coefficient is always greater than that of the first. In the case of a higher viscosity over the entire range of variation, the frequency $\omega$ of the real part of the wave numbers $\operatorname{Rek}_{2,1} \geq \operatorname{Rek}_{2,2}$, and the phase velocity $\mathrm{C}_{\mathrm{y}}$ with increasing $\omega$ tends to infinity.

\section{References}

[1] Padilla, F., de Billy, M. and Quentin, G. (1999) Theoretical and Experimental Studies of Surface Waves on Solid-Fluid Interfaces When the Value of the Fluid Sound Velocity Is Located between the Shear and the Longitudinal Ones in the Solid. The Journal of the Acoustical Society of America, 106, 666-673. 
https://doi.org/10.1121/1.427084

[2] Glorieux, C., Van de Rostyne, K., Nelson, K., Gao, W., Lauriks, W. and Thoen, J. (2001) On the Character of Acoustic Waves at the Interface between Hard and Soft Solids and Liquids. The Journal of the Acoustical Society of America, 110, 1299-1306. https://doi.org/10.1121/1.1396333

[3] Zhu, J., Popovics, J.S. and Schubert, F. (2004) Leaky Rayleigh and Scholte Waves at the Fluid-Solid Interface Subjected to Transient Point Loading. The Journal of the Acoustical Society of America, 116, 2101-2110. https://doi.org/10.1121/1.1791718

[4] Bayon, A., Gascon, F. and Nieves, F.J. (2005) Estimation of Dynamic Elastic Constans from the Amplitude and Velocity of Rayleigh Waves. The Journal of the Acoustical Society of America, 117, 3469-3477. https://doi.org/10.1121/1.1898663

[5] Safarov, I.I., Teshaev, M.Kh. and Akhmedov, M.Sh. (2018) Free Oscillations of a Toroidal Viscoelastic Shell with a Flowing Liquid. American Journal of Mechanics and Applications, 6, 37-49. http://www.sciencepublishinggroup.com/j/ajma

[6] Safarov, I.I. and Boltayev, Z.I. (2018) Methods for Assessing the Seismic Resistance of Subterranean Hydro Structures under the Influence of Seismic Waves. American Journal of Physics and Applications, 6, 51-62.

http://www.sciencepublishinggroup.com/j/ajpa

[7] Safarov, I.I., Teshayev, M.Kh., Boltayev, Z.I. and Akhmedov, M.Sh. (2017) Damping Properties of Vibrations of Three-Layer VIscoelastic Plate. International Journal of Theoretical and Applied Mathematics, 3, 191-198.

[8] Gazis, D.C. (1959) Three-Dimension Investigation of the Propagation of Waves in Hollow Circular Cylinders. II. Numerical Results. The Journal of the Acoustical Society of America, 31, 573-578. https://doi.org/10.1121/1.1907754

[9] Rosenberg, R.L. and Thurston, R.N. (1977) Relationship between Plate and Surface Modes of a Tube. The Journal of the Acoustical Society of America, 61, 1499. https://doi.org/10.1121/1.381450

[10] Ter-Hakobyan, G.L. (2013) Refinement of the Results of the Effect of the Fluid on the Propagation of Waves in an Elastic Cylindrical Shell. Journal Basic Research, Engineering Sciences, 10, 516-520.

[11] Sorokin, S.V. (1997) Fluid-Structure Interaction and Structural Acoustics. Book of Lecture Notes, Technical University of Denmark, Lyngby, 188 p.

[12] Safarov, I.I., Akhmedov, M.Sh. and Boltayev, Z.I. (2016) The Actual Waves in Layered Media. Lambert Academic Publishing, Saarbrücken, 192s.

[13] Safarov, I.I., Boltayev, Z.I. and Akhmedov, M.Sh. (2016) Properties of Wave Motion in a Fluid-Filled Cylindrical Shell. Lambert Academic Publishing, Saarbrücken, 105 p.

[14] Guz, A.N. (2016) Wave Propagation in a Cylindrical Shell with a Viscous Compressible Fluid. Prikladnaia Mekhanika, 16, 10-20.

[15] Safarov, I.I., Akhmedov, M.Sh. and Boltayev, Z.I. (2015) Dissemination Sinusoidal Waves in of a Viscoelastic Strip. Global Journal of Science Frontier Research: F Mathematics \& Decision Sciences, 15, 39-60.

[16] Safarov, I.I., Teshaev, M.X., Akhmedov, M.Sh. and Rajabov, O. (2017) Distribution Natural Waves on the Viscoelastic Cylindrical Body in Plane Strain State. Case Studies Journal, 6, 1-8. http://www.casestudiesjournal.com

[17] Safarov, I.I., Teshaev, M.X., Akhmedov, M.Sh. and Boltaev, Z.I. (2017) Properties of Wave Motion in a Cylindrical Shell Is Contact with a Viscous Fluid. Case Studies Journal, 6, 9-35. http://www.casestudiesjournal.com 
[18] Safarov, I.I., Teshaev, M.Kh., Boltaev, Z.I. and Nuriddinov, B.Z. (2017) Of Own and Forced Vibrations of Dissipative Inhomogeneous Mechanical Systems. Applied Mathematics, 8, 1001-1015. http://www.scirp.org/journal/am https://doi.org/10.4236/am.2017.87078

[19] Safarov, I.I., Teshaev, M.X., Akhmedov, M.Sh. and Boltaev, Z.I. (2017) Distribution Free Waves in Viscoelastic Wedge with and Arbitrary Angle Tops. Applied Mathematics, 8, 736-745. https://doi.org/10.4236/am.2017.85058

[20] Safarov, I.I., Akhmedov, M.Sh. and Boltayev, Z.I. (2016) Ducting in Extended Plates of Variable Thickness. Global Journal of Science Frontier Research: F Mathematics \& Decision Sciences, 16, 33-66.

[21] Safarov, I.I., Teshaev, M.Kh. and Boltayev, Z.I. (2016) Propagation of Linear Waves in Extended Lamellar Bodies. Lambert Academic Publishing, Saarbrücken, 315 p.

[22] Safarov, I.I., Teshaev, M.H. and Boltaev, Z.I. (2012) Wave Processes in Mechanical Waveguide. Lambert Academic Publishing, Saarbrücken, 217 p.

[23] Bozorov, M.B., Safarov, I.I. and Shokin, Y.I. (1966) Numerical Simulation of Vibrations Dissipative Homogeneous and Heterogeneous Mechanical Systems. Moscow, $188 \mathrm{p}$.

[24] Kayumov, S.S. and Safarov, I.I. (2002) Propagation and Diffraction of Waves in Dissipative-Inhomogeneous Cylindrical Deformable Mechanical Systems. Tashkent, $214 \mathrm{p}$.

[25] Grinchenko, V.T. and Myaleshka, V.V. (1981) Harmonic Waves in Elastic Bodies. Kiev, 284.

[26] Vasin, S.V. and Mikolyuk, V.V. (1983) Free Oscillations Tolerable Cylindrical Shells Separated by a Viscous Fluid Hydro Aeromechanics. And the Theory of Elasticity. No. 3, 108-116.

[27] Beginners, Y.N. (1996) The Study of the Spectra of Natural Frequencies of Cylindrical Shells Containing a Compressible Fluid. Science, $74 \mathrm{p}$. 